Journal of Computer Science 8 (5): 775-779, 2012

ISSN 1549-3636

(C) 2012 Science Publications

\title{
An Efficient Method for Contrast Enhancement in Still Images using Histogram Modification Framework
}

\author{
${ }^{1}$ Ravichandran, C.G. and ${ }^{2}$ V. Magudeeswaran \\ ${ }^{1}$ RVS College of Engineering and Technology, Dindigul, Tamilnadu, India \\ ${ }^{2}$ Department of ECE, Anna University of Technology Madurai, Tamilnadu, India
}

\begin{abstract}
Problem statement: Image enhancement improves an image appearance by increasing dominance of some features or by decreasing ambiguity between different regions of the image. Histogram based image enhancement technique is mainly based on equalizing the histogram of the image and increasing the dynamic range corresponding to the image. Approach: Histogram Equalization is widely used in different ways to perform contrast enhancement in images. As a result, such image creates side-effects such as washed out appearance and false contouring due to the significant change in brightness. In order to overcome these problems, mean brightness preserving Histogram Equalization based techniques have been proposed. Generally, these methods partition the histogram of the original image into sub histograms and then independently equalize each sub histogram with Histogram Equalization. Results: The comparison of recent histogram based techniques is presented for contrast enhancement in low illumination environment and the experiment results are collected using low light environment images. Conclusion: The histogram modification algorithm is simple and computationally effective that makes it easy to implement and use in real time systems.
\end{abstract}

Key words: Contrast enhancement, histogram equalization, dynamic histogram equalization, histogram modification, still images, image enhancement, contrast image

\section{INTRODUCTION}

Image enhancement is one of the main areas in digital image processing. The main purpose of image enhancement is to bring out details that are hidden in an image, or to increase the contrast in a low contrast image. It produces an output image that subjectively looks better than the original image by changing the pixel's intensity of the input image. Image enhancement can also be used to provide a better input for other automated image processing systems. It is used as a preprocessing step in medical image processing, speech recognition, texture synthesis and many other image/video processing applications.

A very popular technique for contrast enhancement of images is Histogram Equalization (HE) (Gonzalez and Woods, 2008; Torre et al., 2005). HE is a technique commonly used for image contrast enhancement, since $\mathrm{HE}$ is computationally fast and simple to implement. HE performs its operation by remapping the gray levels of the image based on the probability distribution of the input gray levels.

Brightness preserving Bi-Histogram Equalization (BBHE) (Kim, 1997), Recursive Mean Separate HE (RMSHE) (Chen and Ramli, 2003a), Dynamic
Histogram Equalization (DHE) (Abdullah-Al-Wadud $e t$ al., 2007) and Brightness preserving Dynamic Histogram Equalization (BPDHE) (Kong and Ibrahim, 2008) are the variants of $\mathrm{HE}$ based contrast enhancement techniques. BBHE divides the input image histogram into two parts based on the mean of the input image and then each part is equalized independently. This method tries to overcome the problem of brightness preservation. RMSHE (Chen and Ramli, 2003b) is an improved version of BBHE. However, it is also not free from side effects. In order to deal with above problem, proposed a Dynamic Histogram Equalization (DHE) technique (AbdullahAl-Wadud et al., 2007). However, DHE does not consider the preserving of brightness. For this purpose, Ibrahim and Kong proposed Brightness Preserving Dynamic Histogram Equalization (BPDHE) (Kong and Ibrahim, 2008). This method partitions the image histogram based on the local maxima of the smoothed histogram. It then assigns a new dynamic range to each partition. Finally the output intensity is normalized to make the mean intensity of the resulting image equal to the input one. In this study, we propose a new method based on Histogram modification scheme that works well with still images 
and it enhances the images without making any loss in image details.

\section{MATERIALS AND METHODS}

Histogram Equalization: Histogram is defined as the statistical probability distribution of each gray level in a digital image. Histogram Equalization (HE) is a very popular technique for contrast enhancement of images (Kim and Paik, 2008; Sengee and Choi, 2008). Contrast of images is determined by its dynamic range, which is defined as the ratio between the brightest and the darkest Apixel intensities. The histogram provides information for the contrast and overall intensity distribution of an image. Suppose input image $f(x, y)$ composed of discrete gray levels in the dynamic range $[0, \mathrm{~L}-1]$ then the transformation function $\mathrm{C}\left(\mathrm{r}_{\mathrm{k}}\right)$ is defined as Eq. 1:

$$
\mathrm{S}_{\mathrm{k}}=\mathrm{C}\left(\mathrm{r}_{\mathrm{k}}\right)=\sum_{\mathrm{i}=0}^{\mathrm{k}} \mathrm{p}\left(\mathrm{r}_{\mathrm{i}}\right)=\sum_{\mathrm{i}=0}^{\mathrm{k}} \frac{\mathrm{n}_{\mathrm{i}}}{\mathrm{n}}
$$

where, $0 \leq \mathrm{s}_{\mathrm{k}} \leq 1$ and $\mathrm{k}=0,1,2, \ldots, \mathrm{L}-1$

In Eq. 1, $n_{i}$ represents the number of pixels having gray level $r_{i}, n$ is the total number of pixels in the input image and $\mathrm{P}\left(\mathrm{r}_{\mathrm{i}}\right)$ represents as the probability Density Function (PDF) of the input gray level $r_{i}$. Based on the PDF, the Cumulative Density Function (CDF) is defined as $\mathrm{C}\left(\mathrm{r}_{\mathrm{k}}\right)$. This mapping in (1) is called Histogram Equalization (HE) or Histogram Linearization. Here $s_{k}$ can easily be mapped to the dynamic range of $[0$, L-1] multiplying it by (L-1). However, HE produces an undesirable checkerboard effects on enhanced images (Kim and Paik, 2008). Another problem of this method is that it also enhances the noises in the input image along with the image features.

\section{Limitations in histogram equalization:}

- The Histogram Equalization technique does not take the mean brightness of an image into account

- The HE technique may result in over enhancement and saturation artifacts due to the stretching of the gray levels over the full gray level range

- Histogram equalization can be found on the fact that the brightness of an image can be changed after the histogram equalization

- Nevertheless, HE is not commonly used in consumer electronics such as TV because it may significantly change the brightness of an input image and cause undesirable artifacts

- It can be observed that the mean brightness of the histogram-equalized image is always the middle gray level regardless of the input mean

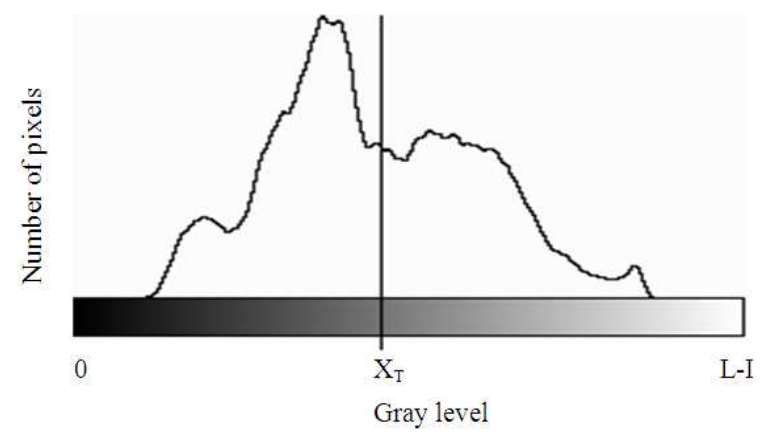

Fig. 1: Bi-histogram equalization. The histogram with range from 0 to $\mathrm{L}-1$ is divided into two parts, with separating intensity $\mathrm{X}_{\mathrm{T}}$. This separation produces two histograms. The first histogram has the range of 0 to $X_{T}$, while the second histogram has the range of $X_{\mathrm{T}+1}$ to L-1

Brightness preserving bi-histogram equalization: In order to overcome the limitations of $\mathrm{HE}$, several brightness preserving methods have been proposed (Kim et al., 2001; Chen and Ramli, 2003a; Sun et al., 2005; Chen et al., 2006; Wang and Ward, 2007; Sengee and Choi, 2008; Wang and Ye, 2005; Agaian et al., 2007).

One of the popular brightness preserving methods is the mean Brightness preserving $\mathrm{Bi}$ Histogram Equalization (BBHE) introduced by Kim (Chen and Ramli, 2003b).

At the beginning, the BBHE divides the original histogram into two sub-histograms based on the mean brightness of the input image as shown in Fig. 1. One of the sub image is set of samples less than or equal to the mean whereas the other one is the set of samples greater than the mean. In this method, the separation intensity $X_{T}$ is presented by the input mean brightness value, which is the average intensity of all pixels that construct the input image. After this separation process, these two histograms are independently equalized by HE. Consequently, the mean brightness can be preserved because the original mean brightness is retained.

Recursive mean-separate histogram equalization: Another description of the BBHE, called Recursive Mean-Separate Histogram Equalization (RMSHE) proposed (Chen and Ramli, 2003a; Rajavel, 2010). This method recursively separates the histogram into multi sub-histograms instead of two sub-histograms as in the BBHE. Initially, two sub-histograms are formed based on the mean brightness of the original histogram. Subsequently, the mean brightness from the two subhistograms obtained earlier is used as the second and third separating points in creating more sub-histograms. In a similar fashion, the algorithm is executed recursively until the desired numbers of sub-histograms are met. Then, the $\mathrm{HE}$ approach is applied independently on each of the sub- 
histogram. However, no significant enhancement is performed by the RMSHE when the number of divided sub histograms is large.

The methods discussed above are based on dividing the original histogram into several sub-histograms by using either the median or mean brightness. Even though the mean brightness is well conserved by the abovementioned methods, but fails to expand the region of sub-histogram located near to the minimum or maximum value of the dynamic range.

Dynamic histogram equalization: The Dynamic Histogram Equalization (DHE) (Abdullah-Al-Wadud et al., 2007) partitions the original histogram based on local minima without using the mean and median value. In order to eliminate the spikes, a $1 \times 3$ smoothing filter is applied across the image. Then, a new dynamic range is assigned to each sub-histogram based on the original dynamic range and the number of pixels in that subhistogram. Generally, the DHE does not consider the mean brightness preservation. Moreover, the $1 \times 3$ smoothing filter is constructed for brightness preserving. Thus, the DHE may cause saturation and it is insufficient to smooth a noisy histogram. As a result, the local minima will be wrongly misclassified and it increases the complexity of the algorithm.

\section{Brightness preserving dynamic histogram} equalization: The Brightness Preserving Dynamic Histogram Equalization (BPDHE) (Kong and Ibrahim, 2008; Kim and Chung, 2008) is the enhanced version of the DHE. Similarly, a smoothing filter is applied to histogram before the partitioning process is carried out. On the contrary, the BPDHE uses the local maxima as the separating point rather than the local minima. After the $\mathrm{HE}$ is implemented to each sub-histogram, brightness normalization is used to ensure the enhanced mean brightness as a close approximation to the original mean brightness. Although the BPDHE performs well in mean brightness preserving, the ratio for brightness normalization plays an important role. A small ratio value leads to insignificant contrast enhancement. For large ratio (i.e., ratio value more than 1 ), the final intensity value may exceed the maximum intensity value of the output dynamic range. The exceed pixels will be quantized to the maximum intensity value of gray levels and produce intensity saturation problem (in MATLAB environment).

\section{RESULTS}

The quantitative analysis are tabulated in Table 1. Figure 2 shows Simulation results of the original image. Figure 3 and 4 present comparison of discrete entropy and average execution time of various $\mathrm{HE}$ techniques

Entropy: In general, the entropy is a useful tool to measure the richness of the details in the output image. It is given by Eq. 2:

$$
\operatorname{Entropy}(\mathrm{p})=-\sum_{\mathrm{k}=0}^{1-1} \mathrm{p}(\mathrm{k}) \log _{2} \mathrm{P}(\mathrm{k})
$$

Table 1: Discrete entropy and average execution time

\begin{tabular}{lll}
\hline Method & $\begin{array}{l}\text { Discrete } \\
\text { entropy (bits) }\end{array}$ & $\begin{array}{l}\text { Average execution } \\
\text { time }(\mathrm{m} \mathrm{sec})\end{array}$ \\
\hline HE & 5.05 & 223.1 \\
BBHE & 5.01 & 256.7 \\
RMSHE & 4.86 & 283.3 \\
DHE & 5.00 & 383.6 \\
BPDHE & 4.67 & 553.2 \\
\hline
\end{tabular}

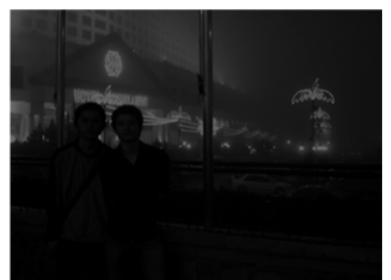

(a)

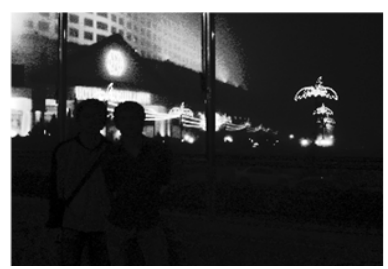

(d)

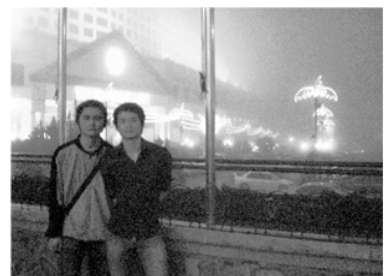

(b)

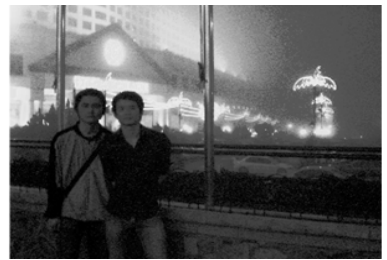

(e)

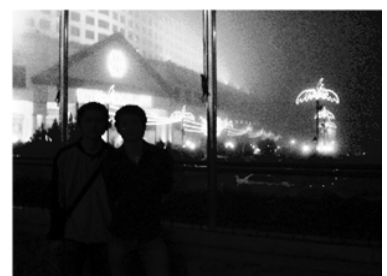

(c)

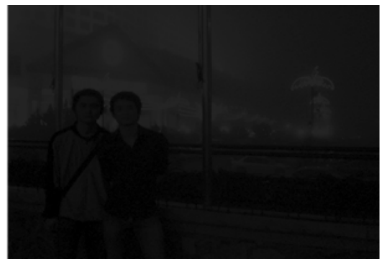

(f)

Fig. 2: Simulation results of the image $(640 \times 840)$. (a) Original image, (b) HE-ed image, (c) BBHE-ed image, (d) RMSHE-ed image, (e) DHE-ed image, (f) BPDHE-ed image 


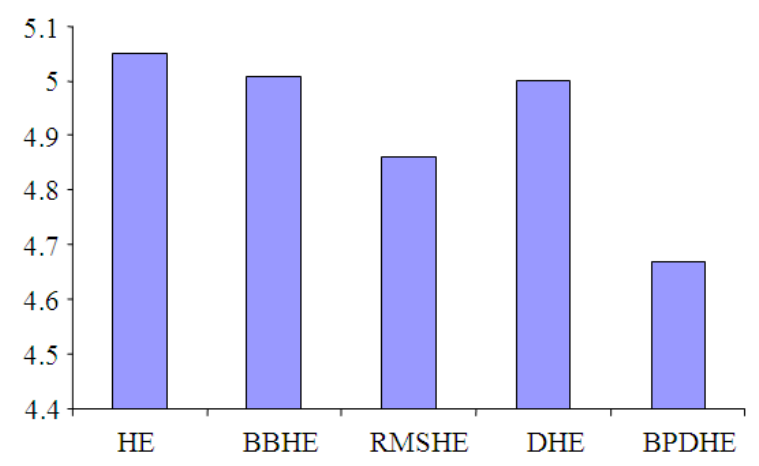

Fig. 3: Comparison of discrete entropy of various HE techniques

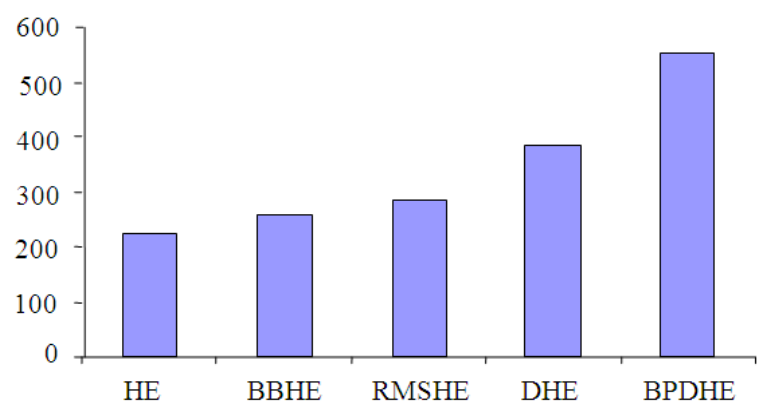

Fig. 4: Comparison of average execution time of various $\mathrm{HE}$ techniques

\section{DISCUSSION}

From Table 1 it is observed that the BPDHE and RMSHE produce lower entropy values than HE. However, the BPDHE produces unnatural enhanced image, while the RMSHE method produces insignificant enhancement to the resultant image. Figure $2 b$ shows that $\mathrm{HE}$ provides a significant improvement in image contrast. However, it also amplifies the noise level of the images along with some artifacts and undesirable side effects such as washed out appearance. Figure $2 \mathrm{c}$ and d shows that the BBHE and RMSHE methods which produce unnatural and insignificant enhancement on the human objects. However, it also has unnatural look because of over enhancement in brightness. The results of DHE and BPDHE show that they do not prevent the washed-out appearance in overall image due to the significant change in brightness. The result of BPDHE (Fig. 2f) shows that the washed-out appearance and it fails to perform well when applied on low contrast images.

\section{CONCLUSION}

The comparison of recent histogram based techniques are presented for contrast enhancement in low illumination environment. DHE is not free from any severe side effects. BPDHE can preserve the mean brightness better than BBHE and DHE. The results of DHE and BPDHE show that they do not prevent the washed-out appearance in overall image due to the significant change in brightness. The abovementioned contrast enhancement techniques perform well on some images but they can create problems when a sequence of images is enhanced, or when the histogram has spikes, or when a natural looking enhanced image is strictly required. In addition, computational complexity and controllability become an important issue when the goal is to design a contrast enhancement algorithm for consumer products. To overcome these artifacts this study presents a new method for contrast enhancement in still images for better perception based on Global Contrast Enhancement (GCE) Histogram modification algorithm. In summary, our goal is to obtain a visually pleasing enhancement method that has lowcomputational complexity and works well with still images obtained from low illumination environment. Moreover, the histogram modification algorithm is simple and computationally effective that makes it easy to implement and use in real time systems.

\section{REFERENCES}

Abdullah-Al-Wadud, M., M.H. Kabir, M.A.A. Dewan and O. Chae, 2007. A dynamic histogram equalization for image contrast enhancement. IEEE Trans. Consumer Elect., 53: 593-600. DOI: 10.1109/TCE.2007.381734

Agaian, S.S., B. Silver and K.A. Panetta, 2007. Transform coefficient histogram-based image enhancement algorithms using contrast entropy. IEEE Trans. Image Process., 16: 741-758. DOI: 10.1109/TIP.2006.888338

Chen, S.D. and A.R. Ramli, 2003. Contrast enhancement using recursive mean-separate histogram equalization for scalable brightness preservation. IEEE Trans. Consumer Electr., 49: 1301-1309. DOI: 10.1109/TCE.2003.1261233

Chen, S.D. and A.R. Ramli, 2003. Minimum mean brightness error bi-histogram equalization in contrast enhancement. IEEE Trans. Consumer Electr., 49: 1310-1319. DOI: 10.1109/TCE.2003.1261234

Chen, Z.Y., B.R. Abidi and D.L. Page, 2006. Graylevel grouping (GLG): An automatic method for optimized image contrast Enhancement-part I: The basic method. IEEE Trans. Image, 15: 2290-2302. DOI: 10.1109/TIP.2006.875204 
Gonzalez, R.C. and R.E. Woods, 2008. Digital Image Processing. 3rd Edn., Prentice Hall, Upper Saddle River, NJ., ISBN-10: 0130946508, pp: 954.

Kim, J.Y., L.S. Kim and S.H. Hwang, 2001. An advanced contrast enhancement using partially overlapped sub-block histogram equalization. IEEE Trans. Circ. Syst. Video Technol., 11: 475-484. DOI: 10.1109/76.915354

Kim, M. and M. Chung, 2008. Recursively separated and weighted histogram equalization for brightness preservation and contrast enhancement. IEEE Trans. Consumer Electr., 54: 1389-1397. DOI: 10.1109/TCE.2008.4637632

Kim, T. and J. Paik, 2008. Adaptive contrast enhancement using gain-controllable clipped histogram equalization. IEEE Trans. Consumer Electr., $\quad$ 54: $1803-1810 . \quad$ DOI: 10.1109/TCE.2008.4711238

Kim, Y.T., 1997. Contrast enhancement using brightness preserving bi-histogram equalization. IEEE Trans. Consumer Elect., 43: 1-8. DOI: $10.1109 / 30.580378$

Kong, N.S.P. and H. Ibrahim, 2008. Color image enhancement using brightness preserving dynamic histogram equalization. IEEE Trans. Consumer Elect., $\quad$ 54: 1962-1968. DOI: 10.1109/TCE.2008.4711259

Rajavel, P., 2010. Image dependent brightness preserving histogram equalization. IEEE Trans. Consumer Electr., 56: 756-763. DOI: 10.1109/TCE.2010.5505998
Sengee, N. and H. Choi, 2008. Brightness preserving weight clustering histogram equalization. IEEE Trans. Consumer Electr., 54: 1329-1337. DOI: 10.1109/TCE.2008.4637624

Sun, C.C., S.J. Ruan, M.C. Shie and T.W. Pai, 2005. Dnamic contrast enhancement based on histogram specification. IEEE Trans. Consumer Electr., 51: 1300-1305. DOI: 10.1109/TCE.2005.1561859

Torre, A.D.L., A.M. Peinado, J.C. Segura, J.L. PerezCordoba and M.C. Benitez et al., 2005. Histogram equalization of speech representation for robust speech recognition. IEEE Trans. Speech Audio Process., 13: 355-366. $\quad$ DOI: 10.1109/TSA.2005.845805

Wang, C. and Z. Ye, 2005. Brightness preserving histogram equalization with maximum entropy: A variational perspective. IEEE Trans. Consumer Electr., $\quad$ 51: 1326-1324. $\quad$ DOI: 10.1109/TCE.2005.1561863

Wang, Q. and R.K. Ward, 2007. Fast image/video contrast enhancement based on weighted thresholded histogram equalization. IEEE Trans. Consumer Elect., 53: 757-764. DOI: 10.1109/TCE.2007.381756 